УДК 621.396 .96

\title{
Grid Method of Collective Guidance \\ and Controlled Target Distribution \\ of a Group of Missiles in the Interests \\ of Group Air Targets Effective Attack
}

\author{
Igor V. Lyutikov*a, Valeriy V. Zamaraev \\ Georgii Y. Shaydurova and Vladimir A. Kopilova \\ ${ }^{a}$ Siberian Federal University \\ 79 Svobodny, Krasnoyarsk, 660041, Russia \\ ${ }^{b} S R C I A S D$ \\ 32 nab. Afanasiya Nikitina, Tver, Russia
}

Received 26.03.2015, received in revised form 17.11.2015, accepted 29.11.2015

The article discusses solving actual scientific problem destruction of group destruction of group air targets aimed at increasing the efficiency index is improved guidance system, which means that the characteristics of its properties. Denotes the solution proposed by the authors in the form of a twophase grid method of collective guidance and target distribution groups managed in the interests of effective missile attack group air targets, optimal criterion of maximum propability of its destructionthe destruction of all of its elements.

Keywords: methods homing homing stages, target distribution, the group of missiles, group air target.

DOI: $10.17516 / 1999-494 X-2015-8-8-1103-1116$.

(c) Siberian Federal University. All rights reserved

* Corresponding author E-mail address: lyutikovigor@mail.ru 


\title{
Сеточный метод коллективного наведения
}

и управляемого целераспределения группы ракет

на групповую воздушную цель,

оптимальный по критерию максимума вероятности

её уничтожения

\author{
И.В. Лютиков ${ }^{a}$, В.В. Замараев ${ }^{6}$, \\ Г.Я. Шайдуров ${ }^{\text {a }}$ В.А. Копылов ${ }^{\text {a }}$ \\ ${ }^{a}$ Сибирский федеральный университет \\ Россия, 660041, Красноярск, Свободньй, 79

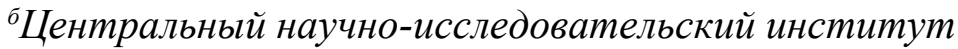 \\ Воздушно-космической оборонь \\ Россия, Тверь, наб. Афанасия Никитина, 32
}

В статье рассматривается решение актуальной научной проблемы уничтожения групповых воздушных иелей, направленной на увеличение показателя эффективности улучшаемой системы наведения, а значит, характеристики её свойства. Обозначено решение в виде предлагаемого авторами двухэтапного сеточного метода коллективного наведения и управляемого иелераспределения группы ракет в интересах эффективной атаки групповой воздушной цели, оптимального по критерию максимума вероятности её уничтожения уничтожения всех её элементов.

Ключевые слова: методы наведения, этапы наведения, целераспределение, группа ракет, групповая воздушная иель.

\section{Актуальность}

Как известно [1-3], основной формой тактического применения истребителей ВВС при ведении борьбы со средствами воздушного нападения противника (CBH) с целью их уничтожения является воздушный бой, три основных этапа которого подразделяются таким образом:

1) дальнее наведение (взлёт, построение группы, полёт в район выполнения боевой задачи): вывод истребителя в такое положение относительно цели, из которого обеспечивается её обнаружение, опознавание и атака;

2) действия в районе выполнения боевой задачи:

- поиск, обнаружение, опознавание воздушной цели многофункциональной (МФ) РЛС, выполнение манёвра для занятия исходного положения для атаки или продолжения сближения с целью;

- атака, которая начинается после захвата цели на автосопровождение МФ РЛС и представляет собой стремительное сближение с целью в сочетании с наведением ракет при их полёте к цели (самонаведения истребителя);

- выход из атаки; начинается после применения вооружения.

3) полёт на аэродром базирования.

Основным видом современного воздушного боя считается дальний всеракурсный групповой воздушный бой [1-3], в котором с одной из сторон участвует от двух до четырёх истреби- 
телей в звене. При действии нескольких противоборствующих истребителей и прикрываемого объекта воздушного базирования в строю на интервалах и дистанциях в пределах одного разрешаемого объёма РЛС по параметрам принимаемых сигналов воздушная цель определяется локатором как единая отметка - групповая воздушная цель (ГВЦ). По мере увеличения расстояния в параметрическом пространстве между принимаемыми отраженными от элементов ГВЦ сигналами до значения, превышающего разрешающую способность РЛС хотя бы по одному из параметров, ГВЦ начинает наблюдаться раздельно $[2,4,5]$.

В процессе уничтожения СВН противника на этапе дальнего наведения истребителя на воздушную цель, в том числе ГВЦ, основной задачей для обеспечения успешного её уничтожения является выведение истребителя в заданное относительно цели положение, соответствующее зоне возможных атак, т.е. в область пространства, из которого возможны самонаведение и прицеливание истребителя с последующим применением вооружения, например ракет с импульсно-доплеровскими полуактивными головками самонаведения (ГСН) [6], представляющих собой элементы многопозиционного авиационного радиолокационного комплекса (МПАРЛК) [7, 8] с «подсветом» из одной точки, например ведущего истребителя. Для занятия тактически выгодного положения для эффективной атаки одиночной ВЦ с вероятностью её уничтожения не ниже требуемой используются известные методы наведения, в том числе в тех или иных разновидностях $[3,5,9]$ :

1. «Кривая атаки», или «Погоня».

2. «Параллельное сближение».

3. «Перехват», или «Прямое сближение», или «Наведение в наивыгоднейшую упреждённую точку встречи».

4. «Манёвр», или «Комбинированный на основе «Прямого сближения» и «Кривой атаки».

5. «Пропорциональное наведение».

6. «Пропорциональное наведение со смещением».

Использование вышеизложенных существующих методов при наведении нескольких одиночных ракет на ГВЦ, наблюдаемую в момент пуска ракет не раздельно, а как одну ВЦ в разрешаемом объёме (при этом ракеты будут наводиться на энергетический центр ГВЦ [1]), неизбежно приведёт к снижению вероятности уничтожения всей ГВЦ - вероятности уничтожения всех её элементов в отдельности. Физика вполне очевидна, так как, во-первых, в подобной ситуации априорно неизвестно, сколько элементов ГВЦ $N_{g v c}$, а значит, априорно неизвестно оптимальное потребное количество запускаемых по ГВЦ ракет $N_{r}$ (или одной ракеты-носителя с разделяющимися на терминальном участке её полёта боевыми самонаводящимися $N_{r}$ элементами, существенно меньшими для цели её гарантированного уничтожения с вероятностью не ниже требуемой. Во-вторых, даже если количество пущенных ракет по ГВЦ зафиксировать с гипотетическим априорно ожидаемым эмпирически полученным запасом, например, равным ожидаемому максимальному количеству противоборствующих истребителей в звене и прикрываемых ими объектов в строю - элементам ГВЦ $N_{r}=N_{g v c}$ ожи , то в данной постановке, если за время полёта ракеты до ГВЦ условия её наблюдения на истребителе, с которого были произведены пуски, не изменятся (при этом ГВЦ так и будет наблюдаться не раздельно, а «слитно» в разрешаемом объёме), целераспределение подлетающих к ГВЦ ракет будет происходить неза- 
висимо на борту каждой ракеты, не координированно, а по случайному закону (так как ракеты наводились на энергетический центр ГВЦ [1]), а значит, и исход атаки ГВЦ будет непредсказуем, вероятность уничтожения ГВЦ - всех элементов ГВЦ - снизится вплоть до вероятности практически невозможного события.

Использование метода «Наведение на упреждённую зону пуска», или «Прямая атака», при организации эффективной атаки ГВЦ на этапе дальнего самонаведения требует от истребителя сближения с зоной применения оружия и вход в зону пуска с требуемыми параметрами наведения по реальной цели [1]. При этом при входе в зону пуска должны обеспечиваться условия разрешения элементов ГВЦ, следовательно, управляемого эффективного целераспределения для дальнейшей координированной атаки, но при этом сам истребитель-«носитель» «подставляется» под вероятный удар атакуемой цели, так как в подобной постановке зона эффективного разрешения элементов ГВЦ с борта истребителя и зона пуска ракет находятся в пределах зоны действия средств поражения атакуемой ГВЦ. Создается дуэльная ситуация, исход которой непредсказуем.

Таким образом, анализ существующих и перспективных методов самонаведения позволяет вскрыть объективные противоречия в практике и теории, которые необходимо устранить, решив актуальную научную задачу (проблему) по разработке оптимального метода наведения группы из $N_{r}$ ракет на групповую воздушную цель из $N_{g v c}$ её элементов для их уничтожения.

Целью данной статьи является изложение концепции решения актуальной научной задачи (проблемы), направленной на увеличение показателя эффективности, а значит, характеристики свойства улучшаемой системы уничтожения ГВЦ и обозначение пути её решения: разработать сеточный метод коллективного наведения и управляемого целераспределения группы ракет в интересах эффективной атаки групповой воздушной цели, оптимального по критерию максимума вероятности её уничтожения - уничтожения всех её элементов.

Разработка метода наведения как решения оптимизационной задачи (проблемы) осуществлена в следующей последовательности [10]:

1) постановка (общая и формализация) задачи (проблемы):

- описание объекта и предмета исследования - метода наведения;

- выбор показателя эффективности как характеристики свойства улучшаемого объекта исследования - системы наведения, формулировка целевой функции - функции качества, определение варьируемых (управляемых) параметров;

- математическая формулировка критерия оптимальности - правила выбора точки оптимума в исследуемой целевой функции;

2) построение математической (имитационной) модели предмета исследования (практически реализована в среде Matlab);

3) решение экстремальной задачи с помощью метода оптимизации [11] (покоординатного подъёма по управляемым параметрам и др.), нахождение координаты точки оптимума.

\section{Постановка научной задачи (проблемы)}

Формализация научной задачи (проблемы) представлена в (1). В качестве критерия выбран условный максимум показателя эффективности системы наведения - вероятности уничтожения ГВЦ $P_{\substack{\text { destruct } \\ \Gamma B Ц}}$ при заданных ресурсных ограничениях $C_{0}$ : 


$$
\begin{aligned}
& K: \max _{\substack{\vec{d}_{1 m} \in D_{1 m} \\
m \subset M}} P_{\substack{\text { destruct } \\
m B L}}\left(\vec{d}_{1 m}, m / \vec{d}_{2 m}, \vec{a}, \vec{e}\right) \\
& \text { при } \\
& C_{m}<C_{o} \text {, }
\end{aligned}
$$

где $P_{\text {destruct }}-$ вероятность уничтожения групповой воздушной цели;

$D_{1 m}^{\Gamma B \amalg}$ область допустимых значений $\vec{d}_{1 m}$;

$m=\overline{1, M}-$ номер варианта метода наведения;

$M$ - количество вариантов методов наведения, среди которых осуществлялся поиск максимума показателя эффективности в соответствии с критерием оптимальности;

$$
\vec{d}_{1 m}=\left[N_{r}\left(N_{g v c}\right), \omega_{n_{g v c} n_{r}}, \vec{P}_{n_{r}}\left(t, t_{0}, \vec{P}_{n_{g v c}}\right)\right]-\text { вектор варьируемых (управляемых) пара- }
$$

метров $m$-го варианта метода наведения

где $n_{r}=\overline{1, N_{r}}-$ номер наводимой на ГВЦ ракеты;

$N_{r}$ - количество наводимых на ГВЦ ракет;

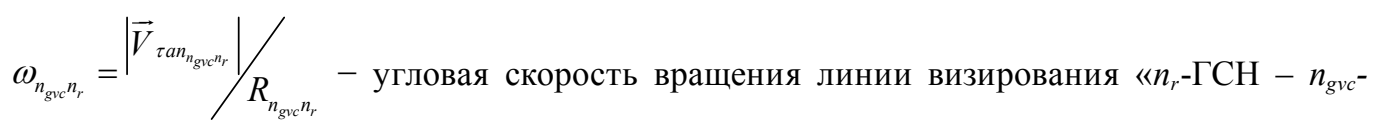

ГВЦ», рад/с;

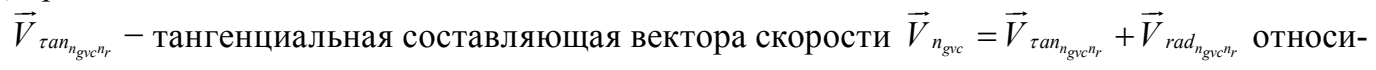
тельно $n_{r}$-ГСН;

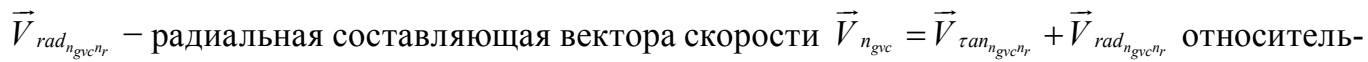
но $n_{r}-\Gamma \mathrm{CH}$;

$R_{n_{g v e} n_{r}}-$ длина линии визирования « $n_{r}-Г \mathrm{CH}-n_{g v c}-\Gamma \mathrm{ВЦ»;}$

$\left.\vec{P}_{n_{r}}=\underset{\text { dal }}{\left[\vec{P}_{n_{r}}\right.}\left(t, t_{0}, \vec{V}_{n_{r}}, \vec{P}_{n_{g v c}}\right), \underset{\text { term }_{n_{r}}}{\vec{P}_{(}}\left(t, t_{0}, \vec{V}_{n_{r}}, \vec{P}_{n_{g v c}}\right)\right]-$ кинематический закон движения $n_{r}$ ракеты при её наведении на ГВЦ, включая этап дальнего (по общегрупповым параметрам) наведения $\underset{{ }_{n}}{\vec{P}_{r l}}\left(t, t_{0}, \vec{V}_{n_{r}}, \vec{P}_{n_{g v c}}\right)$ и этап индивидуального наведения на терминальном участке $\vec{P}_{n_{r}}\left(t, t_{0}, \vec{V}_{n_{r}}, \vec{P}_{n_{\text {gvc }}}\right)$

$t_{0}$ - начальный момент соответствующего этапа наведения;

$\vec{V}_{n_{r}}$ - вектор скорости $n_{r}$-ракеты с ГСН относительно начала координат;

$\Delta R_{\min }$ - минимальный текущий промах наводимой на элемент ГВЦ ракеты с ГСН, $\Delta R_{\min } \leq \Delta R_{0}$

$\Delta R_{0}$ - допустимый промах наводимой на элемент ГВЦ ракеты с ГСН (определяется характеристикой боевой части ракеты - радиусом гарантированного поражения элемента ГВЦ);

$\vec{d}_{2 m}$ - вектор неварьируемых (неуправляемых) параметров $m$-го варианта метода наведения; 
$\vec{a}=\left[N_{g v c}, \vec{P}_{n_{g v c}}\right]-$ вектор параметров, характеризующих противника:

$\underset{g v c}{\rightarrow}$ количество элементов ГВЦ;

$P_{\eta_{g v c}}=\left[t, t_{0}, V_{n_{g v c}}\right]-$ кинематический закон движения $n_{g v c}$ элемента ГВЦ;

$V_{n_{g v c}}-$ вектор скорости $n_{g v c}$ элемента ГВЦ относительно начала координат;

$e$ - вектор параметров, характеризующих среду;

$C_{m}$ - ресурсные ограничения для реализации $m$-го варианта метода наведения $\left(N_{r_{m}}-\right.$ количество ракет, используемых при атаке по ГВЦ, временные (в том числе $t_{z a l p_{m}}-$ время залпа), вычислительные, энергетические затраты);

$C_{0}$ - заданные ресурсные ограничения (заданные $N_{r_{0}}-$ количество ракет, используемых при атаке по ГВЦ, временные (в том числе $t_{z a l p_{0}}$ - время залпа), вычислительные, энергетические затраты).

\section{Построение математической (имитационной) модели}

Изложим условия математического моделирования системы наведения группы ракет с ГСН на ГВЦ. Рассмотрим общие принципы предлагаемого метода наведения группы ракет на групповую цель на примере одного из кинематических вариантов геометрии системы.

Отличия разрабатываемого метода от существующего метода параллельного сближения: 1) наводится не одна ракета, а группа; 2) метод включает два этапа: наведение по общегрупповым параметрам (начальный этап - дальнее коллективное наведение); переход на наведение по индивидуальным параметрам (второй этап - терминальное индивидуальное наведение).

Определению на начальном этапе подлежат: задание ракетам порядка пуска, мест в боевом порядке - строю атакующей группы (мест, организованных в виде узлов сетки на оптимальных интервалах и дистанциях, обеспечивающих при переходе на этап индивидуального наведения благоприятные условия для эффективного целераспределения на основе анализа элементов матрицы угловых скоростей вращения линий визирования « $n_{r}$ ракета с ГСН $-n_{g v c}$ элемент ГВЦ»), условия перехода на индивидуальное наведение (критерий управляемого целераспределения). Исследованию и выбору на первом этапе подлежат параметры, характеризующие этап наведения, информационное обеспечение метода, его структура, взаимосвязи.

Второй этап. Переход на индивидуальное наведение. Осуществляется в соответствии с первоначальными установками и по получаемой на каждой ракете информации о состоянии элементов групповой цели. Метод может рассматриваться с позиций двух вариантов управления: централизованного и децентрализованного. Исследованию и выбору на втором этапе подлежит проработка информационного обеспечения принимаемых решений, структура и программно-алгоритмическое обеспечение метода, выбор параметров.

Метод на обоих этапах наведения целесообразно подвергнуть исследованию в условиях «подсвета» ГВЦ как из одной, так и из нескольких точек - активных бортовых РЛС истребителей из звена, что должно дать преимущество при решении задач эффективного управляемого целераспределения на участке терминального наведения ракет с ГСН на основе усовершенствованных процедур оптимальной внутри- и межпозиционной обработки сигналов [7], отражённых от элементов ГВЦ, в том числе интенсивно-маневрирующих $[12,13]$, для минимизации промаха по ним, что, очевидно, должно благоприятно повлиять на увеличение вероятности уничтожения всей ГВЦ. 
Модель варианта атаки с использованием предлагаемого метода на завершающей стадии его этапа дальнего коллективного наведения (начального этапа) « $N_{r}=2$ ракет с ГСН на $N_{g v c}=2$ элемента ГВЦ» с использованием «подсвета» со своего ведущего истребителя (ВИ) иллюстрирует рис. 1. В точках ГСН, ГВЦ в глобальной системе координат визуализировано векторное

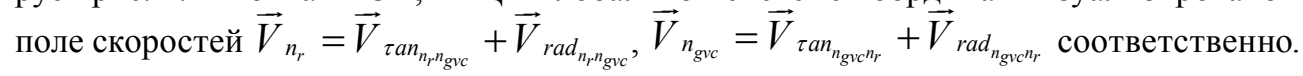

Рассмотрим момент пуска ведущей (первой) ракеты с ГСН. В этот момент вектор её скорости должен быть направлен в интересующую исследователя упреждённую точку (УТ) встречи ракеты с ГВЦ (по общегрупповому её параметру, например, ведущего элемента ГВЦ или центр группы).

В этой точке (УТ) пересекается прямолинейная траектория ведущей ракеты и прямолинейная траектория ведущего элемента ГВЦ. В любой промежуточный момент линия, соединяющая ведущую ракету с целью (линия визирования), остаётся параллельна исходной «Пуск ГСН - Пуск ГВЦ». Дальнее коллективное наведение реализуется по принципу параллельного сближения ведущей ракеты с ведущим элементом ГВЦ. Остальные ракеты с ГСН держат строй относительно ведущей ракеты на заданных оптимальных интервалах и дистанциях до момента перехода на этап индивидуального наведения.

В постановке показатель эффективности - вероятность уничтожения $N_{g v c}$ (всех) целей. Ресурсные ограничения - количество ракет, используемых при атаке (не больше заданного $N_{r_{0}}$ ракет), а также $t_{z a l p_{m}}$ - время залпа ракет при атаке. Очевидно, что $t_{z a p_{m}}$ целесообразно ограничить для минимизации ожидаемого ущерба в случае ответной атаки. Важно определить варьируемые (управляемые) параметры и оценить их влияние на выбранную целевую функцию. При поиске решения поставленной задачи (проблемы), а именно в момент перехода от этапа дальнего коллективного наведения по общегрупповым параметрам к этапу индивидуального наведения, выбирается такой вариант алгоритма целераспределения и его параметры (стратегия целераспределения), которые удовлетворяют ранее описанному критерию оптимизации

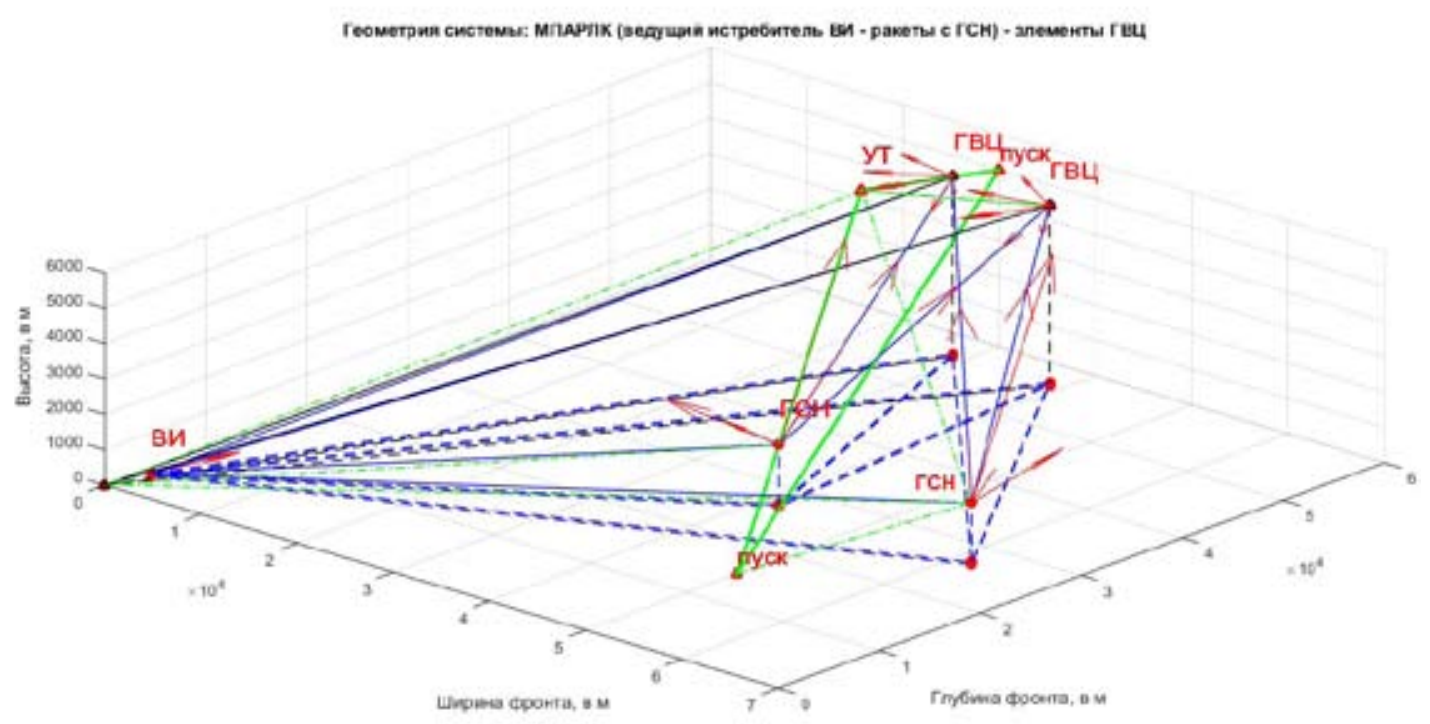

Рис. 1 Модель варианта атаки « $N_{r}=2$ ракет с ГСН на $N_{g v c}=2$ элемента ГВЦ» 


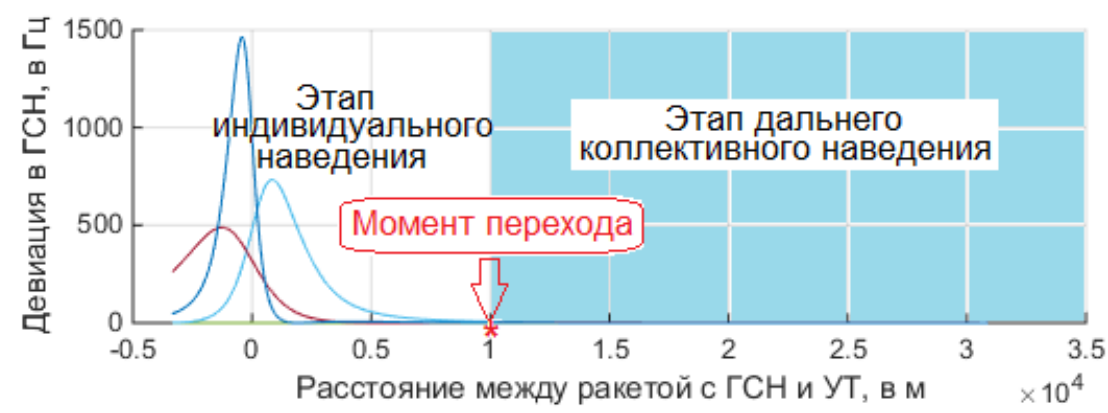

Рис. 2. Зависимость девиации частоты $\Delta f_{n_{r} n_{g v c}}$ принимаемого в ГСН ракеты сигнала на различных этапах наведения

и который обеспечивает максимальную вероятность уничтожения всей групповой цели при заданных ресурсных ограничениях (на ракеты и временные ограничения) при заданных исходных данных.

Протяжённость терминального этапа индивидуального наведения ракет с ГСН при их залпе с максимальной дальности пуска 200-300 км (для современных и перспективных ракет) существенно меньше, чем протяжённость этапа их дальнего коллективного наведения. Как показывают предварительные расчеты и оценки на модели, момент перехода на этап индивидуального наведения составляет 10 км от атакуемой ГВЦ (рис. 2).

Как видно на рис. 2, при рассмотренном кинематическом варианте геометрии системы (рис. 1) на удалении от элементов ГВЦ на 10 км и менее появляются зависимости параметров принимаемых в ГСН ракет сигналов, в частности девиации частоты, что создаёт предпосылку для их дальнейшего разрешения и повышения степени согласованности их обработки, что, в свою очередь, благоприятно скажется на уменьшении ошибок в индивидуальном наведении на элементы ГВЦ и их эффективном поражении.

\section{Правило выбора оптимального варианта}

алгоритма целераспределения

\section{и его параметров (стратегии целераспределения).}

Критерий целераспределения

Известно [1-5, 12-15], что по мере сближения наблюдателя (например, $n_{r}$-ракеты с ГСН) с ВЦ (например, с $n_{g v c}$-элементом ГВЦ), находящейся на дальности $R_{n_{g v c} n_{r}}$, тангенциальная составляющая скорости $\vec{V}_{\text {tan }_{n_{g v c} n_{r}}}$ растёт и, соответственно, увеличивается частотная девиация $d f_{n_{g c v^{n},}} / d t$ (2) принимаемого на фиксированной длине волны $\lambda$ отраженного сигнала от ВЦ:

$$
\frac{d f_{n_{g v c} n_{r}}}{d t}=\frac{\left|\vec{V}_{\operatorname{\tau an}_{n_{g v c} n_{r}}}\right|^{2}}{R_{n_{g v c} n_{r}} \lambda}=\omega_{n_{g v c} n_{r}}\left|\vec{V}_{\operatorname{\tau an}_{n_{g v c n_{r}}}}\right| / \lambda .
$$

В условиях дальнего коллективного наведения по общегрупповым параметрам критерий параллельного сближении в упреждённую точку (УТ) встречи соблюдается лишь для ведущей ракеты с ГСН на ведомый элемент ГВЦ. При этом в локальной системе координат ГСН $n_{r}=1$ 
ведущей ракеты, как инерциальной системе отсчёта, тангенциальная составляющая вектора скорости $n_{g v c}=1$ ведущего элемента ГВЦ (3), угловая скорость вращения линии визирования $\ll n_{r}=1$ ведущая ракета с ГСН $-n_{g v c}=1$ ведущий элемент ГВЦ» (4) и девиация частоты наблюдаемого в $n_{r}=1$ ведущей ракете с ГСН сигнала, отраженного от $n_{g v c}=1$ ведущего элемента ГВЦ (5), равны нулю:

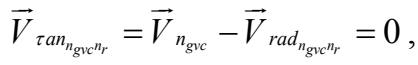

$$
\begin{aligned}
& \omega_{n_{g v c} n_{r}}=\left|\vec{V}_{\tau^{2 a n_{n_{g v c} n_{r}}}}\right| / R_{n_{\text {gvc }} n_{r}}=0, \\
& \frac{d f_{n_{g v c} n_{r}}}{d t}=\omega_{n_{g v c} n_{r}}\left|\vec{V}_{\tau^{2} a n_{n_{g v c} n_{r}}}\right| / \lambda=0 .
\end{aligned}
$$

Для остальных ведомых ракет с ГСН, оптимальным образом держащих строй на установленных интервалах и дистанциях в соответствии с предлагаемым методом, критерий параллельного сближения на этапе дальнего коллективного их наведения, очевидно, не выполняется, и из модели (рис. 1) это видно. Вследствие этого и учитывая (2), при сокращении дистанции между группой ракет с ГСН и ГВЦ увеличивается влияние ракурсных зависимостей, что приводит к появлению угловых скоростей линий визирования, отличных от линии « $n_{r}=1$ ведущая ракета с ГСН $-n_{g v c}=1$ ведущий элемент ГВЦ». Например, для случая наведения « $N_{r}=2$ ракет с ГСН на $N_{g v c}=2$ элемента ГВЦ» это такие линии, как: 1$) \ll n_{r}=1$ ракета с ГСН $-n_{g v c}=2$ элемент ГВЦ»; 2) « $n_{r}=2$ ракета с ГСН $-n_{g v c}=1$ элемент ГВЦ»; 3) « $n_{r}=2$ ракета с ГСН $-n_{g v c}=2$ элемент ГВЦ».

Обозначим случайные независимые при управляемом целераспределении события:

$A_{n_{r} n_{g v c}}$ - уничтожение $n_{r}$-ракетой $n_{g v c}$-элемент ГВЦ.

При наведении « $N_{r}$ ракет с ГСН на $N_{g v c}$ элементов ГВЦ» полная группа несовместных событий [12] имеет вид

$$
\sum_{n_{g v c=1}}^{N_{g v c}} P_{d e s t r u c t}=\sum_{n_{g v c}=1}^{N_{g v c}} \sum_{n_{r}=1}^{N_{r}} P\left(A_{n_{r} n_{g v c}}\right)=1 .
$$

Вероятность $P_{\substack{d e s t r u c t \\ \Gamma B Ц}}(7)$ уничтожения всей ГВЦ вычисляется как вероятность произведения [12] вероятностей $P_{\substack{d e s t r u c t \\ n_{\text {gve }}}}$ независимых случайных событий - уничтожения каждого $n_{g v c}$-элемента ГВЦ и находится по формуле

$$
P_{\text {destruct }}=\prod_{n_{g v c}=1}^{N_{g v c}} P_{\text {destruct }}=\prod_{n_{g v c}=1}^{N_{g v c}} \sum_{n_{r}=1}^{N_{r}} P\left(A_{n_{r} n_{g v c}}\right)
$$

Величины $P\left(A_{n_{r} n_{g v}}\right)$ для всех значений $n_{r}$ и $n_{g v c}$ можно представить в виде матрицы размерностью $\left[N_{r}, N_{g v c}\right](8)$. 


$$
[P(A)]=\left[\begin{array}{l}
P\left(A_{11}\right) \ldots P\left(A_{1 n_{g v c}}\right) \ldots P\left(A_{1 N_{g v c}}\right) \\
\ldots \\
P\left(A_{n_{r} 1}\right) \ldots P\left(A_{n_{r} n_{g v c}}\right) \ldots P\left(A_{n_{r} N_{g v c}}\right) \\
\ldots \\
P\left(A_{N_{r} 1}\right) \ldots P\left(A_{N_{r} n_{g v c}}\right) \ldots P\left(A_{N_{r} N_{g v c}}\right)
\end{array}\right]
$$

Ей соответствуют матрицы (9-12) той же размерности по измеряемым параметрам [14] принимаемого сигнала в точке местоположения ГСН ракеты - матриц исходной информации.

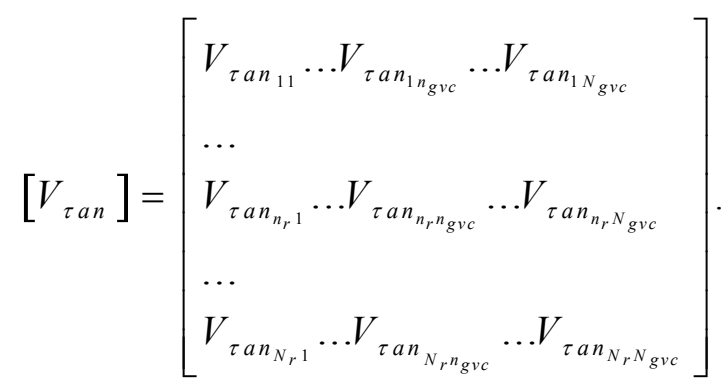

$$
\begin{aligned}
& {[\omega]=\left[\begin{array}{l}
\omega_{11} \ldots \omega_{1 n_{g v c}} \ldots \omega_{1 N_{g v c}} \\
\ldots \\
\omega_{n_{r} 1} \ldots \omega_{n_{r} n_{g v c}} \ldots \omega_{n_{r} N_{g v c}} \\
\ldots \\
\omega_{N_{r} 1} \ldots \omega_{N_{r} n_{g v c}} \ldots \omega_{N_{r} N_{g v c}}
\end{array}\right]} \\
& {[\Delta R]=\left[\begin{array}{l}
\Delta R_{11} \ldots \Delta R_{1 n_{g v c}} \ldots \Delta R_{1 N_{g v c}} \\
\ldots \\
\Delta R_{n_{r} 1} \ldots \Delta R_{n_{r} n_{g v c}} \ldots \Delta R_{n_{r} N_{g v c}} \\
\ldots \\
\Delta R_{N_{r} 1} \ldots \Delta R_{N_{r} n_{g v c}} \ldots \Delta R_{N_{r} N_{g v c}}
\end{array}\right]} \\
& {[\Delta f]=\left[\begin{array}{l}
\Delta f_{11} \ldots \Delta f_{1 n_{g v c}} \ldots \Delta f_{1 N_{g v c}} \\
\ldots \\
\Delta f_{n_{r} 1} \ldots \Delta f_{n_{r} n_{g v c}} \ldots \Delta f_{n_{r} N_{g v c}} \\
\ldots \\
\Delta f_{N_{r} 1} \ldots \Delta f_{N_{r} n_{g v c}} \ldots \Delta f_{N_{r} N_{g v c}}
\end{array}\right]}
\end{aligned}
$$


Из физики процесса наведения очевидно, что для уничтожения всей ГВЦ, а именно каждого из её элементов в отдельности, достаточно воспользоваться при управляемом целераспределении одной из «диагоналей» матрицы (8), назовём данный выбор стратегией управляемого целераспределения. Подобное правило, очевидно, избавит от ситуаций дублирования целераспределения, события станут зависимыми от выбора, которые могли бы привести в случае ограниченного количества ракет $N_{r_{0}}$ используемых при атаке ГВЦ к снижению $P_{d e s t r u c t}$. Очевидно также, что та из стратегий целераспределения, что даёт максимальное значение $P_{\text {destruct }}$, и будет оптимальной стратегией управляемого целераспределения в соответствии с критерием в постановке.

Для наглядности рассмотрим вариант целераспределения « $N_{r}=4$ ракет с ГСН на элемента ГВЦ», геометрическая интерпретация произведений случайных событий при котором поясняется на рис. 3. В соответствии с (7) в случае выбора в качестве стратегии управляемого целераспределения главной диагонали матрицы (8) вероятность уничтожения ГВЦ находим по формуле (13) как вероятность произведения $N_{g v c}=4$ независимых случайных событий:

$$
P_{\text {destruct }}=\prod_{n_{g v c}=1}^{N_{g v c}} P\left(A_{n_{g v c} n_{g v c}}\right)=P\left(A_{11} A_{22} A_{33} A_{44}\right) .
$$

Ввиду того что на практике априорно узнать безусловные вероятности событий (8) не представляется возможным, как варианты вполне возможно при управляемом целераспределении использовать «диагонали» матриц исходной информации (измеряемых параметров) (9-12). На примере использования матрицы (12) оптимальной стратегией управляемого целераспределения при этом будет являться та «диагональ», которую можно представить в виде векторастроки размерностью $\left[1, N_{g v c}\right]$, элементы которой имеют индексы назначения: $\left[n_{r_{\min }}, n_{g v c_{\min }}\right]-$ индексы «минимальных» элементов матрицы (12), и находятся по правилу (14) - критерию целераспределения, что предположительно, и это (вполне очевидно из физики процесса наведения и согласованной обработки сигнала с минимальной ЛЧМ $[12,16])$ уменьшит ошибки

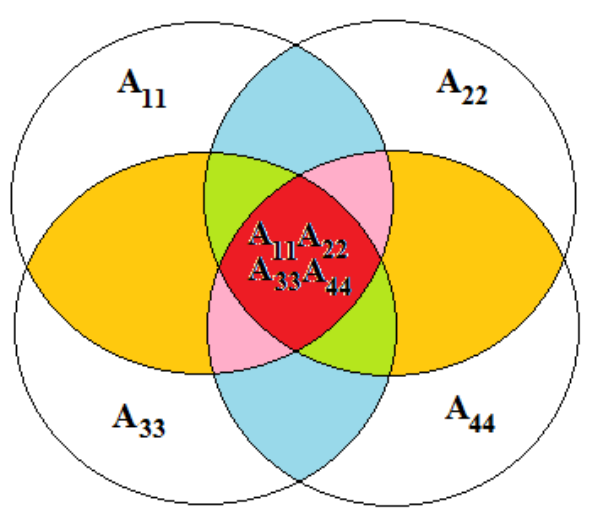

Рис. 3. Произведение случайных событий при наведении « $N_{r}=4$ ракет с ГСН на $N_{g v c}=4$ элемента ГВЦ» 
в индивидуальном наведении на элементы ГВЦ, что даст максимальное значение $P_{d e s t r u c t}$, а значит, и $\underset{\text { destruct }}{P_{\text {ГВЦ }}}(1,7)$ :

$$
\begin{aligned}
& K_{\substack{o p t \\
\lfloor P}}: \\
& {\left[n_{r_{\min }}, n_{g v c_{\min }}\right]=\min _{\substack{n_{g v c}=1, N_{g v c} \\
n_{r}=1, N_{r}}}([\Delta f]), n_{r_{o p t}}=\overline{1, N_{r}}, n_{g v c_{o p t}}=\overline{1, N_{g v c}} .}
\end{aligned}
$$

Условимся под матрицей управляемого целераспределения (назначения) (CTDM) понимать матрицу размером $\left[N_{r}, N_{g v c}\right]$, элемент которой равен 1 , если $n_{r_{\text {opt }}}$-ракета с ГСН назначена на $n_{g v c_{\text {opt }}}$-элемент ГВЦ. В противном случае значение элемента назначения равно нулю. Задача управляемого целераспределения - решить, каким элементам присвоить единицы.

Назначая цели ракетам в соответствии с критерием (14), получим (15):

$$
\operatorname{CTDM}\left(n_{r_{o p t}}, n_{g v c_{o p t}}\right)=\left\{\begin{array}{l}
1, \text { если } \Delta f\left(n_{r}, n_{g v c}\right)=\min _{\substack{n_{g v c}=1, N_{g v c} \\
n_{r}=1, N_{r}}}([\Delta f]) \\
0, \text { ecлu } \Delta f\left(n_{r}, n_{g v c}\right) \neq \min _{\substack{n_{g v c}=\overline{1, N_{g v c}} \\
n_{r}=1, N_{r}}}([\Delta f])
\end{array}, n_{r_{o p t}}=\overline{1, N_{r}}, n_{g v c_{o p t}}=\overline{1, N_{g v c}},\right.
$$

или учитывая (14)

$$
\operatorname{CTDM}\left(n_{r_{o p t}}, n_{g v c_{o p t}}\right)=\left\{\begin{array}{l}
1, \text { еслu }\left[n_{r_{o p t}}, n_{g v c_{o p t}}\right]=\left[n_{r_{\min }}, n_{g v c_{\min }}\right] \\
0, е с л u\left[n_{r_{o p t}}, n_{g v c_{o p t}}\right] \neq\left[n_{r_{\min }}, n_{g v c_{\min }}\right]
\end{array}, n_{r_{o p t}}=\overline{1, N_{r}}, n_{g v c_{o p t}}=\overline{1, N_{g v c}} .\right.
$$

Таким образом, принцип заполнения матрицы (15), (16), оптимального выбора ракетой цели, заключается в том, что $n_{r_{o p t}}$-ракета выбирает тот $n_{g v c}$-элемент ГВЦ, у которого текущая девиация (элемент матрицы (12)) принимаемого на этой ракете сигнала минимальна. При этом каждое последующее назначение ракеты на элемент ГВЦ осуществляется посредством поиска «минимального» элемента матрицы (12) по принципу выборки «минимального» элемента матрицы «без возврата» [17]. Это значит, что последующий шаг поиска «минимального» элемента осуществляется внутри блочной матрицы, получаемой путем слияния блоков матрицы (12) на предыдущем шаге, за исключением строки и столбца, соответствующих назначенным $\left[n_{r_{o p t}}\right.$, $\left.n_{g c_{\text {opt }}}\right]$ на предыдущем шаге.

Таким образом, использование при управляемом целераспределении в качестве матрицы исходных данных матрицы девиаций частоты (12) принимаемых от элементов ГВЦ сигналов увеличит степень согласованности их дальнейшей обработки $[12,16]$, а значит, уменьшит потери, обусловленные несогласованностью приёма, в конечном счёте создаст условия для уменьшения текущего промаха, что, очевидно, благоприятно скажется на увеличении (7), что требует дальнейшего подтверждения имитационным моделированием с использованием метода Монте-Карло. От целераспределения до эффективности атаки остается пройти путь - наведение каждой $n_{r_{\text {opt }}}$-ракеты с ГСН на $n_{g v c}$ opt -элемент ГВЦ и подрыв БЧ (в модели достаточно довести

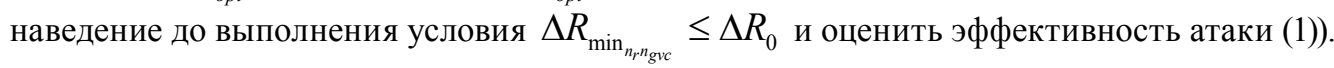

В перспективе возможно исследовать влияние на показатель эффективности (1) принципов как централизованного (с использованием обмена и обработки информации [7, 8] между 
истребителями из звена и атакующими ракетами в группе), так и децентрализованного (с ключевой ролью как ведущей ракеты, так и автономного) целераспределений, в том числе основанных на методах роевого интеллекта [18] для управления группами наводимых на элементы ГВЦ ракет.

\section{Список литературы}

[1] Богданов А.В., Филонов А.А., Ковалев А.А. и др. Методы самонаведения истребителей и ракет класса воздух-воздух на групповую воздушную иель. Красноярск: СФУ, 2014 [Bogdanov A.V., Filonov A.A., Kovalev A.A. Methods homing fighters and missiles of class air-to-air aerial target group. Krasnoyarsk, SibFU, 2014]

[2] Лютиков И.В., Замараев В.В., Кучин А.А. и др. Успехи современной радиоэлектроники, 2014, 5, 47-52 [Lutikov I.V., Zamaraev V.V., Kuchin A.A. The successes of modern Radioelectronics, 2014, 5, 47-52]

[3] Лютиков И.В., Замараев В.В., Кучин А.А. и др. Журнал СФУ. Техника и технологии, 2014 7(8), 911-918 [Lutikov I.V., Zamaraev V.V., Kuchin A.A. J. Sib. Fed. Univ. Eng. technol., 2014 7(8), 911-918]

[4] Богомолов Н.П., Сидоров В.Г., Шайдуров Г.Я., Лютиков И.В. Радиотехника, 2013, 6, 43-45 [Bogomolov N.P., Sidorov V.G., Shaidurov G.Ia., Lutikov I.V. Radiotechnics, 2013, 6, 43-45]

[5] Богданов А.В., Кучин А.А., Мещеряков В.Г. и др. Радиотехника, 2013, 6, 65-69 [Bogdanov A.V., Kuchin A.A., Meshcheriakov V.G. Radiotechnics, 2013, 6, 65-69]

[6] Замараев В.В., Кучин А.А., Лютиков И.В. Успехи современной радиоэлектроники, 2012, 9, 107-110 [Zamaraev V.V., Kuchin A.A., Lutikov I.V. The successes of modern Radioelectronics, 2012, $9,107-110]$

[7] Замараев В.В., Лютиков И.В. Радиотехника, 2008, S130, 77-83 [Zamaraev V.V., Lutikov I.V. Radiotechnics, 2008, S130, 77-83]

[8] Канащенков А.И. и др. Облик перспективных бортовых радиолокационных систем. Возможности и ограничения. М.: ИПРЖР, 2002 [Kanashchenkov A.I. Look promising airborne radar systems. Possibilities and limitations. Moscow, IPRZhR, 2002]

[9] Дудник П.И., Ильчук А.Р., Татарский Б.Г. Многофункциональные радиолокаиионные cuстемы: учеб. пособие /ред. Б.Г. Татарский. М.: Дрофа, 2007. 283 с. [Dudnik P.I., Il'chuk A.R., Tatarskii B.G. Multifunctional radar system. Moscow, Drofa, 2007, 283 p.]

[10] Черных М.М., Богданов А.В., Буров А.С. и др. Вестник МГТУ им. Н.Э.Баумана. Сер. Приборостроение, 1999, 4, 16-26 [Chernykh M.M., Bogdanov A.V., Burov A.C. Bulletin of the Bauman Moscow State Tech. Univ. Instrumentation, 1999, 4, 16-26]

[11] Радиоэлектронные системы. Основы построения и теория. Справочник. Изд. 2-е, перераб. и доп. / ред. Я.Д. Ширман. М.: Радиотехника, 2007, 512 с. [Electronic systems. Bases of construction and theory. Ia.D.Shirman (ed.) Moscow, Radiotechnics, 2007, 512 p.]

[12] Вентцель Е.С. Теория вероятностей: учебник. М.: Академия, 2003, 576 c. [Venttsel' E.S. Probability theory. Moscow, Akademiia, 2003, 576 p.]

[13] Рубан А.И. Методы оптимизации: учеб. пособие. Красноярск: ИПЦ КГТУ, 2004, 528 с. [Ruban A.I. Optimization methods. Krasnoyarsk State Techn. Univ. 2004, 528 p.]

$$
-1115-
$$


[14] Пантелеев А.В., Летова Т.А. Методы оптимизации в примерах и задачах: учеб. пособие. М.: Высш. шк., 2005. 544 с. [Panteleev A.V., Letova T.A. Optimization methods in examples and problems. Moscow, Vyssh. Shk., 2005. 544 p.]

[15] Вексин С.И. Обработка радиолокаџионных сигналов в доплеровских головках самонаведения. М.: Изд-во МАИ, 2005. 244 с. [Veksin S.I. Processing of radar signals in the Doppler homing, Moscow Aviation Institute, 2005. 244 p.]

[16] Авиачия ПВО России и научно-технический прогресс: боевые комплексы и системы вчера, сегодня, завтра: монография / ред. Е.А. Федосов. М.: Дрофа, 2004 [Air defense aviation of Russia and scientific technical progress. Combat complexes and systems yesterday, today, tomorrow. E.A. Fudosov (ed.). Moscow, Drofa, 2004]

[17] Ярлыков М.С., Богачев А.С., Меркулов В.И., Дрогалин В.В. Радиоэлектронные комплексы навигации, прищеливания и управления вооружением летательных аппаратов. Т. 1. Теоретические основы / ред. М.С. Ярлыков. М.: Радиотехника, 2012 [Iarlykov M.S., Bogachev A.S., Merkulov V.I., Drogalin V.V. Radio electronic navigation systems, sighting and fire control aircraft. Vol. 1. Theoretical foundations. Moscow, Radiotechnics, 2012]

[18] Иванов Д.Я. Известия Южного федерального университета. Технические науки, 2011, 116(3) [Ivanov D.Ia. Izvestiya SFedU. Engineering Sciences, 2011, 116(3)] 